\section{$\mathbf{m} / \mathbf{s}$}

médecine/sciences $1992 ; 8: 782-3$

\title{
ENCORE L'HÉMOGLOBINE, TOUJOURS L'HÉMOGLOBINE
}

\section{Jean Rosa}

\section{ADRESSE}

J. Rosa : professeur. Inserm U. 91, CHU Henri-Mondor, 51, avenue du Maréchal-deLattre-de-Tassigny, 94010 Créteil, France.

TIRÉS A PART hémoglobine a été l'une des grandes vedettes des années 1950 qui ont vu, à son propos, apparaître la première anomalie moléculaire chez l'homme: Pauling [1] montre en 1949 que les globules rouges drépanocytaires contiennent une hémoglobine de charge anormale; puis Ingram [2] en 1956 découvre que dans cette hémoglobine une valine est remplacée par un acide glutamique. Dans les années 1960, sont établies la séquence des chaînes d'hémoglobine, puis, presque simultanément, leurs structures dans l'espace (Perutz [3]). Un immense travail de détection et d'identification des mutants s'en est suivi qui a permis la découverte de plus de 600 variants structuraux fournissant tous les modèles théoriques prévisibles : mutations ponctuelles, doubles mutations, délétions, insertions, chaînes raccourcies ou rallongées. Ces travaux ont très fortement stimulé les recherches technologiques aboutissant entre autres à l'électroisofocalisation (IEF) et au développement d'une stratégie d'étude des mutations par chromatographie qui a débouché sur des méthodes chromatographiques à hautes performances (HPLC). Quand celles-ci furent couplées aux méthodes modernes de spectrométrie de masse, on devint capable d'identifier un mutant en quelques couples d'heures - au lieu des mois auparavant nécessaires - et des modifications post-traductionnelles très nombreuses dont beaucoup affectent probablement d'autres protéines que l'hémoglobine [4]. Bel exemple de l'évolution des sciences, au moment même où l'optimalisation des techniques d'analyse des protéines faisait diminuer de plusieurs ordres de grandeur les quantités de matériel nécessaires et les délais requis, les techniques des recombinants d'ADN surgissaient qui rendent les premières presque désuètes. La génétique moléculaire naissante permit en effet une "percée d'Avranches "* dans le domaine des thalassémies, de leur classification et de leurs mécanismes. On soupçonnait l'existence de plusieurs mutations thalassémiques. La génétique moléculaire permit d'en trouver plus d'une centaine, illustration de cette extrême diversité de mutations, ce qui semble être la règle en pathologie génétique. Avoir la séquence du gène de structure, la séquence des protéines codées ne règle pas pour autant le problème du traitement des maladies génétiques comme ont fait semblant de le croire pendant quelque temps, dans la fièvre de l'innovation, les tenants de la nouvelle génétique, de la génétique inverse, maintenant plus justement dénommée "génétique positionnelle". Une fois encore le modèle hémoglobinique doit servir d'exemple. Il y a plus de 30 ans que la séquence protéique est connue, plus de 25 ans que ses messagers ont été isolés, plus de 15 ans que les gènes ont été clonés et, cependant, les problèmes thérapeutiques demeurent. Thalassémies (et drépanocytose) peuvent certes bénéficier du traitement radical constitué par les greffes de

\footnotetext{
* Pour les non-historiens, percée du front de Normandie par le général Patton en juillet 1944, ayant entrainé la rupture du front allemand de l'Ouest.
} 
moelle, mais celles-ci ne sont pas sans risques, imposent la quête aléatoire d'un donneur compatible et sont l'apanage des pays riches, certes de plus en plus atteints par ces maladies - qui restent cependant surtout fréquentes dans les pays du tiers-monde. D'où l'intérêt considérable suscité par des travaux comme ceux évoqués dans les articles d'Yves Beuzard et de Josée Pagnier (p. 784 et 790 de ce numéro). Le problème des modèles animaux est abordé dans le premier article ( $p$. 784). Ceux-ci se sont en effet révélés indispensables aux progrès dans la compréhension des mécanismes physiopathologiques des thalassémies et de la drépanocytose, ainsi que dans la tentative de leur correction. L'hémolyse est reconnue depuis longtemps comme l'accident majeur des thalassémies : les chaînes d'hémoglobines "célibataires ", en raison de la non-synthèse des chaînes adverses, précipitent sur la membrane érythrocytaire. On ne sait cependant toujours pas par quel mécanisme cette précipitation induit l'hémolyse : reconnaissance de la membrane altérée au niveau de la rate? oxydation des thiols de la membrane (ou d'enzymes) par libération d'ions superoxydes ou par création de disulfides ? L'absence de modèle animal possédant les hémoglobines humaines empêchait de tester ces hypothèses. La création de souris thalassémiques, puis la réussite de greffes de gènes de globine humaine s'exprimant fortement chez la souris [5] ouvrent d'immenses perspectives dans ce domaine. On peut dès lors suivre aisément le devenir des chaînes greffées, expérimenter l'effet d'éventuels inducteurs de synthèses telle l'érythropoïétine. Le cas de la drépanocytose est encore plus surprenant. Il était tenu pour établi que la maladie - ô combien pléiotropique dans ses accidents ! - était tout bonnement due à la polymérisation intraglobulaire de la désoxy-Hb $\mathrm{S}$ qui entraînait falciformation et thrombose [6]. Las, la réalisation de souris $\mathrm{S} / \mathrm{S}$ a montré que les choses sont loin d'être aussi simples, ces souris n'étant pas malades. Toute une recherche est donc en cours pour obtenir des souris malades, à l'aide de super-Hb $\mathrm{S}$ sur le modèle de l'Hb S-Antilles [7] et produites par mutagenèse dirigée. Cela est en passe d'être réalisé et permettra, d'une part, d'identifier d'éventuels facteurs interagissant avec la mutation $\mathrm{S}$ pour favoriser ou inhiber l'expression de la maladie, et, d'autre part, de disposer avec ces animaux d'irremplaçables outils (que les défenseurs des animaux me pardonnent) pour tester d'éventuelles thérapeutiques concurrentes de la greffe de moelle. Greffes des gènes défaillants bien évidemment (qui aurait cru, il y a quatre ans, que les gènes $\alpha$ et $\beta$ greffés auraient une expression parfaitement coordonnée ?) mais aussi action de différents produits sur l'expression de gènes d'hémoglobine fœtale dont on sait depuis longtemps que la dérépression constituerait une solution thérapeutique. Ces animaux permettront enfin, dans le même ordre d'idées, de tester toutes les substances susceptibles d'inhiber la falciformation. En ce qui concerne ce dernier chapitre, l'arrivée de ces modèles murins de drépanocytose sera inestimable car le problème non résolu des drogues inhibitrices (nombreuses) in vitro est celui de leur métabolisme et de leur toxicité qui, pour d'évidentes raisons éthiques, ne peuvent être testés que sur des animaux. On conçoit aisément que le même chemin devra être suivi pour nombre de maladies génétiques.

L'article de J. Pagnier ( $p .790$ de ce numéro) traite d'un tout autre problème tout aussi passionnant : celui de la production d'un substitut du sang, ce terme étant entendu en tant que transporteur d'oxygène. En effet, on assiste actuellement dans ce domaine à un véritable concert philarmonique associant les plus avancées des techniques de la biochimie des protéines et de la génétique moléculaire. Le problème posé est immense : faire face aux besoins sans cesse croissants en sang alors que redoublent les problèmes de la transfusion sanguine et que s'éloignent les espoirs de pouvoir utiliser des substituts organo-chimiques tels des dérivés fluoro-carbonés qui ont clairement montré leurs limites. La possibilité de faire synthétiser des chaînes d'hémoglobine d'abord à E. Coli puis à la levure a ouvert la voie à une approche radicalement différente. Les problèmes à résoudre actuellement consistent essentiellement dans la production d'une hémoglobine à affinité diminuée pour l'oxygène de manière à contre-balancer l'hyperaffinité de solutions d'hémoglobine injectables obligatoirement plus diluées qu'elles ne le sont dans les globules rouges. Les connaissances acquises par l'étude des corrélations structure/fonction de très nombreux mutants naturels à affinité modifiée pour l'oxygène permettent d'orienter le design de la molécule idéale qui, dès lors, pourra être obtenue par mutagenèse dirigée. Une voie concurrente serait de créer des lignées de gros mammifères transgéniques (non porteurs, on l'espère, de virus humanotropes !) avec le gène bricolé codant pour cette hémoglobine. Quelles possibilités en cas de réussite! Non, décidément non, l'hémoglobine, ce n'est pas fini!

\section{RÉFÉRENCES}

1. Pauling L, Mano HA, Singer SJ, Wells IC. Sickle cell anemia : a molecular disease. Science $1949 ; 110$ : 543-8.

2. Ingram VM. A specific chemical difference between the globins of normal human and sickle cell anaemia haemoglobin. Nature $1966 ; 178$ : 792-4.

3. Perutz MF. The Hb molecule. Sci Amer $1964 ; 211: 64-76$

4. Promé D, Blouquit Y, Ponthus C, Promé JC, Rosa JE. Structure of the human adult hemoglobin minor fraction $A_{1 b}$ by electrospray and secondary ion mass spectrometry. J Biol Chem 1991; 266 : 13050-4.

5. Grosveld F, Van Assendelft GB, Greaves DR, Kollias G. Position-independant, high-level expression of the human $\beta$-globin gene in transgenic mice. Cell 1987; 51 : 975-85.

6. Rosa JE. Un modèle en recherche clinique: la drépanocytose. médecine/sciences 1986 ; 2 : 558-67.

7. Monplaisir N, Mérault G, Poyart C, et al. Hb S-Antilles : a variant with lower solubility than $\mathrm{Hb} \mathrm{S}$ and producing sickle cell disease in heterozygotes. Proc Natl Acad Sci USA 1986 ; 83 : 9363-7. 